\title{
EVOLUCIÓN EN LA FORMA DE SOSPECHA Y EXTENSIÓN DE LA CIRUGÍA DEL CÁNCER DE MAMA EN UN CENTRO DE RADIO-ONCOLOGÍA*
}

\author{
Drs. Verónica López L. ${ }^{1}$, Claudia Carvajal C. ${ }^{1}$, Manuel Gallardo M. ${ }^{2}$, Moisés Russo N. ${ }^{1,3}$ \\ 1 Residente de Radioterapia Oncológica, Instituto de Radiomedicina IRAM. \\ 2 Cirugía en Patología Mamaria, Clínica Santa María. \\ 3 Facultad de Medicina Universidad Diego Portales. \\ Santiago, Chile.
}

\begin{abstract}
Evolution in breast cancer suspicion and extent of surgery at a radio-oncology center
\end{abstract}

Introduction: Breast cancer diagnosis and treatment had evolved over the past quarter century. From self-examination to mammography as main suspicion tool and from radical to conservative surgery plus radiotherapy as prefered treatment. The aim of this review was to assess the evolution of presentation and local management of breast cancer at a Chilean radio-oncology center. Materials and Methods: We analyzed 1.204 breast cancer patients who received postoperative irradiation on two four-years periods. The first period included 223 patients and coincides with the introduction of mammography and conservative surgery. The second included 981 patients managed according to current guidelines. The variables analyzed were type of clinical suspicion, time between clinical suspicion and diagnosis confirmation, type of surgery, histology and tumor size. Data were obtained from medical records and analyzed using STATA 12. Results: In the second period mammographic suspicion reached $39.8 \%$. Time between clinical suspicion and histological diagnosis was reduced to $50 \%$, the proportion of tumors larger than $2 \mathrm{~cm}$ was reduced from 61 to $45 \%$, the proportion of DCIS was tripled from 6 to $18 \%$, use of conservative surgery has an absolute increase of $28 \%$. All of these differences were statistically significant $(\mathrm{p}<0.01)$. Conclusion: The introduction of mammography and conservative management allowed early diagnosis of breast cancer in the analyzed population.

Key words (MeSH): Breast neoplasms, mammography, segmental mastectomy.

\section{Resumen}

Introducción: El enfrentamiento del cáncer de mama evolucionó en el último cuarto de siglo desde el autoexamen a la mamografía como herramienta de sospecha y desde el tratamiento con cirugía radical a la cirugía conservadora más radioterapia. El objetivo de esta revisión fue evaluar la evolución de la presentación

\footnotetext{
Los autores no refieren conflictos de interés.

Correspondencia: Dra. Verónica López L. veritolopezl@gmail.com
}

*Recibido el 15 de noviembre de 2013 y aceptado para publicación el 12 de diciembre de 2013. 
y manejo local del cáncer de mama en un centro de radio-oncología. Materiales y Método: Se analizaron 1.204 pacientes con cáncer de mama que recibieron irradiación post-operatoria en dos períodos de cuatro años. El primer período incluyó 223 pacientes y coincide con la introducción de mamografía y cirugía conservadora. El segundo incluyó 981 pacientes manejadas según el estándar actual. Las variables analizadas fueron: forma de sospecha, tiempo entre sospecha y confirmación diagnóstica, tipo de cirugía, histología y tamaño tumoral. Los datos fueron obtenidos de fichas clínicas y analizados con STATA 12. Resultados: Al comparar el segundo período con el primero se evidenció un aumento absoluto del 39,8\% de la sospecha por mamografía, disminución a la mitad del tiempo entre sospecha y diagnóstico histológico, reducción de la proporción de tumores mayores a $2 \mathrm{~cm}$ de 61 a $45 \%$, triplicación en la proporción de carcinoma ductal in situ de 6 a $18 \%$ y aumento absoluto de $28 \%$ del uso de cirugía conservadora. Todas estas diferencias fueron estadísticamente significativas $(\mathrm{p}<0,01)$. Conclusión: En el grupo analizado la introducción de mamografía y manejo conservador se asoció a un aumento de sospecha y diagnóstico precoz del cáncer de mama.

Palabras clave: Neoplasia mamaria, mamografía, cirugía conservadora.

\section{Introducción}

En 1970 el tratamiento estándar del cáncer de mama era la mastectomía radical modificada. La cirugía conservadora se introdujo en Chile en $1976^{1}$ $\mathrm{y}$ actualmente es el enfoque de elección en el manejo quirúrgico de esta patología. La mamografía, por su parte, ha ido desplazando paulatinamente al autoexamen de mama como método de sospecha precoz ${ }^{2-5}$, por lo que resulta interesante analizar la evolución de la presentación y tratamiento loco-regional del cáncer de mama en Chile en el último cuarto del siglo XX.

Con la hipótesis que la consulta precoz aumentaría por la probabilidad de recibir un tratamiento menos agresivo, se investigó la evolución de la detección del cáncer de mama en Chile durante este período y se evaluó si se asoció a cambios en la extensión final del tratamiento quirúrgico realizado.

El objetivo de este trabajo es evaluar la evolución de la presentación y el manejo quirúrgico del cáncer de mama en un centro radio-oncológico en Chile.

\section{Material y Métodos}

Se realizó una revisión de las fichas clínicas de las pacientes del Instituto de Radio-Medicina (IRAM), un centro de radioterapia chileno creado en 1977 que recibe pacientes tanto de seguros privados como estatales. Se analizaron datos recuperados de las fichas de dos períodos: el período A (1978-1981) y el período B (2001-2004). El Período A coincide con el inicio del uso de la mamografía en $\mathrm{Chile}^{6,7} \mathrm{y}$ la introducción del uso de la cirugía conservadora y el período $\mathrm{B}$ a uno más moderno que incluye pacientes tratados bajo el estándar actual.

Se revisaron 1.204 fichas de pacientes ingresadas por cáncer de mama que recibieron irradiación postoperatoria con intención curativa. Se encontraron 223 pacientes tratadas en el período A y 981 en el período $\mathrm{B}$.
Tres médicos revisores extrajeron de las fichas los siguientes datos: fecha y forma de sospecha (examen físico por profesional de la salud, autoexamen o mamografía), fecha de confirmación diagnóstica (biopsia core o quirúrgica), tipo de cirugía (mastectomía parcial o mastectomía radical modificada), histología (carcinoma infiltrante o Carcinoma ductal in situ (CDIS)) y tamaño tumoral.

Cuando los datos no pudieron ser extraídos de las fichas se excluyeron dichas pacientes de ese análisis específico.

El análisis estadístico fue realizado con STATA 12 utilizando prueba exacta de Fisher y $\chi^{2}$ para las tablas de contingencia y el test de Mann-Whitney para comparación de medianas donde fuese apropiado. Se consideró estadísticamente significativo $\mathrm{p}<0,001$.

\section{Resultados}

Las 1.204 pacientes evaluadas, al momento de la derivación tenían una mediana de edad de 55 años. Un $40 \%$ de estas pertenecían al sistema nacional de salud y $60 \%$ a otro sistema de financiamiento.

En los 1.172 pacientes en que se analizó la forma de sospecha, hubo un aumento estadísticamente significativo de esta a través de mamografía entre el período A con 1 caso $(0,5 \%)$ y el B con $388(40,3 \%)$, con una disminución significativa de la sospecha por autoexamen (Figura 1). La frecuencia de sospecha por examen clínico se mantuvo constante en ambos períodos ( 10,5 y $11,1 \%$ respectivamente).

En cuanto a la histología se analizaron 1.153 fichas y se observó una variación estadísticamente significativa en la proporción de pacientes con CDIS $(\mathrm{p}<0,001)$, aumentando de $6 \%$ a $18 \%$ entre los períodos A y B (Tabla 1). Por el contrario, el diagnóstico de tumores infiltrantes disminuyó de $94 \%$ a $82 \%$ entre ambos períodos $(\mathrm{p}<0,001)$.

El tiempo transcurrido entre sospecha y diagnós- 


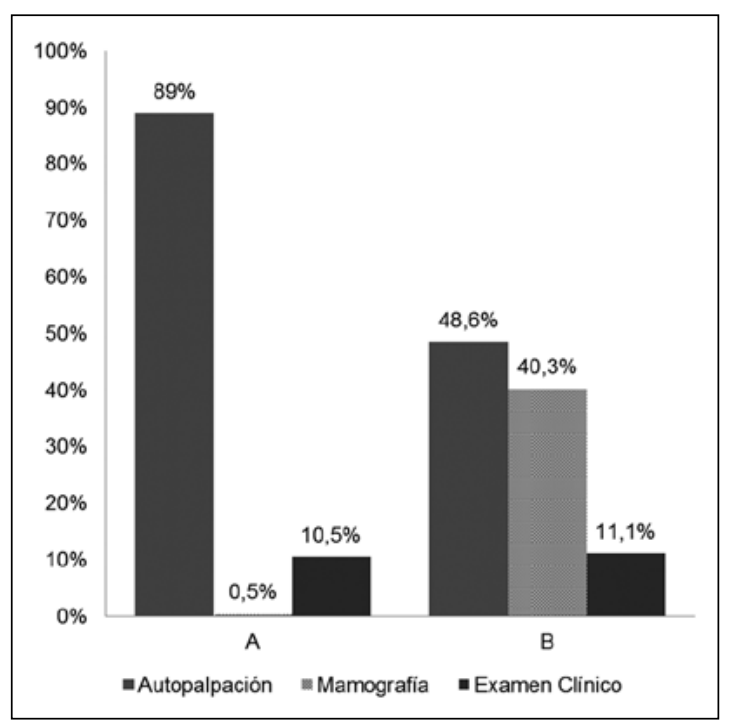

Figura 1. Forma de sospecha diagnóstica según período de tiempo. Evolución en la forma de sospecha diagnóstica (autopalpación, mamografía, examen clínico) según período de tiempo analizado (A y B) expresado en porcentaje de pacientes analizados.

Tabla 1. Tipo de cirugía, histología y tamaño tumoral de ambos períodos

\begin{tabular}{|lcc|}
\hline Característica & A \% (n) & B \% (n) \\
Tipo de cirugía* & & \\
Radical & $48,8(102)$ & $20,6(198)$ \\
Conservadora & $51,2(107)$ & $79,4(764)$ \\
Tipo histológico* & & \\
CDIS & $6,1(11)$ & $18(175)$ \\
Infiltrante & $93,9(169)$ & $82(798)$ \\
Tamaño tumoral* & & \\
$\leq \mathrm{T} 1$ & $39,4(67)$ & $54,6(516)$ \\
$\geq \mathrm{T} 2$ & $60,6(103)$ & $45,4(429)$ \\
\hline
\end{tabular}

*p < 0,001. Período A: de 1978 a 1981, período B: de 2001 a 2004, CDIS: carcinoma ductal in situ. $\leq \mathrm{T} 1: \leq 2 \mathrm{~cm}$, $\geq \mathrm{T} 2$ : $>2 \mathrm{~cm}, \mathrm{n}$ : número. Comparación de ambos períodos A y $\mathrm{B}$ respecto al tipo de cirugía (radical o conservadora), histología (CDIS o infiltrante) y tamaño tumoral (T) expresado en porcentaje y número de pacientes.

tico pudo calcularse para 1.059 pacientes que tenían este dato y la diferencia de las medianas de tiempo fue estadísticamente significativa $(p<0,001)$ entre períodos. La mediana de tiempo entre sospecha y diagnóstico histológico fue de 98 días para el período A (1 día - 97 meses) y para el período B fue de 41 días (1 día-126 meses).

El dato de tipo de cirugía estaba disponible en
1.171 fichas, 209 del período A y 962 del período B. Se observó un aumento en la proporción de pacientes tratadas de forma conservadora (Tabla 1) de $51 \%$ a $79 \%$ entre el período A y B respectivamente, mientras que el $49 \%$ de las pacientes fue tratada con mastectomía radical modificada en el período $\mathrm{A}$, disminuyendo a $21 \%$ en el período $\mathrm{B}(\mathrm{p}<0,001)$.

Hubo una significativa disminución en el tamaño promedio de los tumores (Tabla 1) medido según el informe de biopsia quirúrgica. Con 1.115 fichas donde este dato estaba disponible, 170 del período A y 945 del período $\mathrm{B}$, fue posible ver que la proporción de tumores con tamaño $>$ a $2 \mathrm{~cm}$ disminuyó de $61 \%$ en el período A a $45 \%$ en el período B. En forma opuesta los tumores aumentaron de $39 \%$ a $55 \%$ entre ambos períodos.

\section{Discusión}

Los hallazgos confirmaron la presunción que motivó el estudio: se apreciaron diferencias entre los dos períodos estudiados: aumento de los tumores sospechados por mamografía, acortamiento del tiempo entre sospecha y diagnóstico definitivo, reducción en el tamaño tumoral, aumento en la proporción de CDIS y aumento del uso de cirugía conservadora. Todas estas diferencias fueron estadísticamente significativas.

Debido a los riesgos y costos del sobrediagnóstico de tumores sin importancia clínica el debate en torno a este tema es de particular interés para utilización óptima de recursos de salud en países como Chile ${ }^{8,9}$.

El impacto del aumento de la sospecha mamográfica es difícil de evaluar, pues este trabajo tiene un diseño descriptivo, observacional y transversal. Además, la introducción de la mamografía en este medio fue posterior a la introducción del tratamiento conservador del cáncer de mama. Sin embargo, estudios anteriores realizados en este mismo centro mostraron que las pacientes con sospecha mamográfica pudieron ser tratadas en forma conservadora en un mayor porcentaje ${ }^{2,3}$.

Si bien una de las debilidades de este estudio es que representa la experiencia de un solo centro, el aumento del uso de cirugía conservadora que se aprecia en este reporte se observó también en estudios internacionales y en la actualidad constituye el tratamiento recomendado por la Sociedad Chilena de Mastología para el manejo de cáncer de mama temprano ${ }^{10-13}$.

Tanto en esta serie como en estudios extranjeros se ha observado que las mujeres que se realizan mamografías aumentan la probabilidad que el diagnóstico de cáncer de mama sea hecho en tumores 
menos invasores y de menor tamaño, bajando su probabilidad de ser tratadas con mastectomía9. Las pacientes del segundo período analizado en este estudio demoraron, además, menos tiempo en consultar al médico ante la sospecha de un tumor de mama. Sin embargo, no puede omitirse comentario sobre los enormes intervalos en que algunas de las pacientes dejaron evolucionar su enfermedad sin estudio confirmatorio. Esto se puede deber al sesgo de recuerdo en pacientes que relatan una sospecha por autoexamen de varios años antes de consultar. Las pacientes con tiempos de contemplación mayores a un año fueron un total de 84 , con $76 \%$ de estas pacientes teniendo sospecha por autoexamen.

Así mismo, el porcentaje de diagnóstico de CDIS presenta un aumento significativo cuando la mamografía es utilizada, lo que es esperable por ser un instrumento diagnóstico capaz de pesquisar tumores antes de que estos sean palpables ${ }^{3}$. A pesar de la evidencia que avala el uso de la mamografía para detección precoz de tumores de mama, en la actualidad no existe en Chile un programa nacional de tamizaje. Las recomendaciones médicas deben ser concordantes con la evidencia y es necesario dirigir esfuerzos hacia la implementación de un programa de mayor cobertura que permita seguir aumentando la pesquisa precoz, y por ende, disminuyendo la radicalidad de los tratamientos.

En conclusión, la introducción progresiva de la mamografía significó un aumento de la sospecha y diagnóstico precoz (menor tamaño e invasión) del cáncer de mama entre los 2 períodos estudiados. Esto se asoció a una menor radicalidad de los tratamientos y a un aumento de la proporción de los tumores no infiltrantes confirmando la hipótesis que gatilló el presente estudio.

\section{Agradecimientos}

M. Ramón Baeza, Claudia Castillo, José Gutiérrez, Ana María Salas.

\section{Referencias}

1. Baeza MR, Arraztoa J, Solé J, Rodríguez R. Conservative Surgery Followed by Radical Radiotherapy in the management of stage I carcinoma of the breast. Int. J Radiat Oncol Biol Phys. 1982;8:1775-80.
2. Acevedo JC, Baeza MR, Fernández C, Ibarra I, Solé J, Solé C, y cols. Cáncer de mama diagnosticado en un programa de detección precoz por mamografía (screening). Rev Chil Cancerol y Hematol. 1998;8:27-34.

3. Hepp R, Baeza MR. Tratamiento conservador del carcinoma ductal in situ de la mama. Rev Med Chile 1999;127:1351-6.

4. Moss S, Cuckle H, Evans A, Johns L. Effect of mammographic screening from age 40 years on breast cancer mortality at 10 years' follow-up: a randomised controlled trial. Lancet 2006;368:2053-60.

5. Bleyer A, Welch G. Effect of Three Decades of Screening Mammography on Breast-Cancer Incidence. N Engl J Med. 2012;367:1998-2005.

6. Bengolea P, Parker S: Pioneros en el combate al cáncer mamario. Cosas.com; 2009, 4 de enero. Disponible en: www.cosas.com/index.php/reportajes/234enero-2009/3494-3273 (Consultado el 25 de mayo de 2013).

7. Hospital Militar de Santiago, Nuestra Historia: Los hitos médicos y tecnológicos. Hosmil.cl; Disponible en: www.hosmil.cl/index.php?option=com_content\&view= article \&id=196:nuestra-historia\&catid=37:nuestrohospital\&Itemid=146 (Consultado el 25 de mayo de 2013).

8. Braithwaite D, Zhu W, Hubbard RA, O'Meara ES, Miglioretti DL, Geller B, et al. Screening Outcomes in Older US Women Undergoing Multiple Mammograms in Community Practice: Does Interval, Age, or Comorbidity Score Affect Tumor Characteristics or False Positive Rates? J Natl Cancer Inst. 2013;105:334-41.

9. Woloshin S, Schwartz L. The Benefits and Harms of Mammography Screening Understanding the Trade-offs. JAMA. 2010;303:164-5.

10. Serra I, Daysi Maya G. Cáncer de mama en Chile. Tardanza en su atención médica. Cuadernos Médico Sociales 2011;51:233-9.

11. Advisory Committee of Breast Cancer Screening, England. Screening for breast cancer in England: past and future. J Med Screen. 2006;13:59-61.

12. León A, Droppelmann N, Rencoret C, Cunill E, Vigueras G, Quintas A, y cols. Revisión de la Literatura para Manejo Quirúrgico del Cáncer de Mama en Etapas I, II y IIIa: Aspectos Generales. Medwave 2010;10:44-9.

13. Fisher B, Anderson S, Bryant J, Margolese R, Deutsch $\mathrm{M}$, Fisher E, et al. Twenty-year follow-up of a randomized trial comparing total mastectomy, lumpectomy, and lumpectomy plus irradiation for the treatment of invasive breast cancer. N Eng J Med. 2002;347:123341. 